\title{
Plateforme \\ numérique d'accompagnement professionnel en enseignement: défis liés à son élaboration, à sa mise en œuvre et aux stratégies collaboratives déployées
}

\author{
Auteurs \\ Ugo Collard-Fortin, Doctorant en éducation, Agent de liaison projet \\ TrEnsForma, Université du Québec à Chicoutimi, Canada, \\ ugo.collard-fortin@uqac.ca
}

Andréanne Gagné, professeure, Collaboratrice projet TrEnsForma, Université de Sherbrooke, Canada, andreanne.gagne4@usherbrooke.ca

Sandra Coulombe, professeure titulaire, Coresponsable projet TrEnsForma, Université du Québec à Chicoutimi, Canada, sandra coulombe@uqac.ca

Nadia Cody, professeure titulaire, Coresponsable projet TrEnsForma, Université du Québec à Chicoutimi, Canada, nadia cody@uqac.ca

Thomas Rajotte, professeur, Collaborateur projet TrEnsForma, Université du Québec à Rimouski, Canada,

thomas rajotte@uqar.ca

Manon Doucet, doyenne des études, Coresponsable projet TrEnsForma, Université du Québec à Chicoutimi, Canada, manon doucet@uqac.ca 


\section{REVUE HYBRIDE DE L'ÉDUCATION}

\section{Résumé}

Les compétences du $21^{\mathrm{e}}$ siècle constituent des enjeux de formation devant davantage s'inscrire au cœur de la préparation de la relève enseignante. Parmi ces dernières compétences, la collaboration représente une voie prometteuse permettant certes d'agir positivement sur le développement de compétences en enseignement, mais pouvant également être utilisée comme un levier de développement pour les intervenants de cette formation. Le projet TrEnsForma (Transition pour les Enseignant(e)s en Formation) a vu le jour en s'appuyant sur cette prémisse. Se présentant sous la forme d'une plateforme numérique, ce dispositif d'accompagnement professionnel s'adresse aux futurs et nouveaux diplômés de tous les secteurs d'enseignement et met en œuvre des activités de mentorat, d'autoformation, de coformation ainsi que des ressources audiovisuelles et des formations créditées. TrEnsForma est développé «pour » et «par» la collaboration. À l'invitation du numéro thématique sur les $\mathrm{C} 21$, ce texte illustre les défis du développement (le " par ") de cette plateforme numérique misant sur la collaboration à la fois comme moteur de son élaboration et de sa mise en œuvre. Par le truchement du récit d'expérience, les grandes étapes ainsi que les activités qui ont été nécessaires pour assurer son édification sont décrites, ce qui permet de dégager les stratégies collaboratives qui se sont révélées des leviers tout au long du processus de réalisation du projet.

Mots-clés : collaboration; accompagnement; stratégies collaboratives ; formation à l'enseignement ; plateforme numérique 


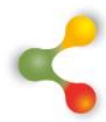

\section{REVUE HYBRIDE DE L'ÉDUCATION}

\section{Introduction}

Dans le cadre de la formation initiale et de l'insertion professionnelle des personnes enseignantes, la collaboration universitémilieu favorise le développement des compétences professionnelles et permet de soutenir les étudiants s'y inscrivant ou y évoluant. Les orientations ministérielles, les écrits portant sur les compétences du $21^{\text {e }}$ siècle et les résultats de recherche portant sur la collaboration sont formels: la collaboration contribue à la professionnalisation des enseignants et à l'amélioration de leurs pratiques pédagogiques. Cet article présente une démarche de développement d'un dispositif, une démarche essentiellement collaborative entre plusieurs universités québécoises et plusieurs partenaires des milieux scolaires œuvrant à la réalisation de cette innovation. À terme, cette innovation offrira aux personnes nouvelles et futures enseignantes des modalités d'accompagnement (mentorat, coformation, etc.) leur permettant de développer leurs savoirs professionnels, dont ceux liés à la collaboration, également soutenus par les référentiels des compétences du $21^{\mathrm{e}}$ siècle.

\section{Intérêt des compétences du $21^{\mathrm{e}}$ siècle et formation à l'enseignement : considérer la collaboration comme dénominateur commun}

À l'échelle internationale, des chercheurs, des décideurs et des acteurs du monde de l'éducation s'intéressent aux compétences du $21^{\text {e }}$ siècle pour former des citoyens, des travailleurs et des dirigeants possédant les connaissances et les attitudes afin d'œuvrer dans une société en changement et hyperbranchée (Bryan et al., 2015; Gouvernement de l'Ontario, 2016 ; Pruneau et al., 2015). Les compétences du $21^{\mathrm{e}}$ siècle englobent moult termes analogues comme compétences de base, compétences essentielles, compétences clés, socle commun de connaissances et de compétences et bien d'autres encore évoquant 1) des sujets de base et des thèmes du $21^{\mathrm{e}}$ siècle, 2) des compétences liées à la créativité, l'innovation, la pensée critique, la résolution de problèmes, la communication et la collaboration ; 3) des compétences liées à l'usage des technologies de l'information et de la communication, et 4) des compétences dites de vie et de carrière : flexibilité, adaptabilité, initiative, autonomie, sociabilité, interculturalisme, productivité, responsabilité, leadership et responsabilité (Anderson, 2010 ; Pruneau et al., 2015 ; Voogt et Roblin, 2012; Westera, 2001). Selon Hart et Ouellet (2013) ainsi qu'une analyse des référentiels de compétences du $21^{\text {e }}$ siècle réalisée par le ministère de l'Éducation de l'Ontario (Gouvernement de l'Ontario, 2016), les compétences liées à la collaboration, la communication et l'usage des technologies sont mentionnées dans tous les cadres de compétences du $21^{\mathrm{e}}$ siècle. 


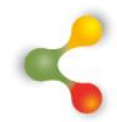

\section{REVUE HYBRIDE DE L'ÉDUCATION}

Cette reconnaissance et cette valorisation des compétences du $21^{\text {e }}$ siècle concernant la capacité d'un individu à collaborer et à se réseauter afin de réaliser différents objectifs, avec ou sans technologies de l'information et des communications, ont été réaffirmées dans le récent référentiel québécois des compétences professionnelles en enseignement (Gouvernement du Québec, 2020). À cet effet, le ministère de l'Éducation et de l'Enseignement supérieur du Québec considère que le travail enseignant doit s'appuyer sur la mise en place d'un travail collaboratif se situant au cœur de l'acte d'enseigner. Plus spécifiquement, il est considéré que l'exercice de la profession enseignante implique la mise en œuvre d'un professionnalisme collaboratif s'appuyant sur deux compétences professionnelles distinctes qui doivent être déployées et développées dans le cadre de la formation initiale en enseignement, soit les compétences : s'impliquer activement au sein de l'équipe-école et collaborer avec la famille et les partenaires communautaires.

L'expression et la mobilisation de ces compétences, dites relationnelles et sociales, appellent la mise en place d'une culture véritable de collaboration et d'entraide. Celles-ci concernent l'ensemble des principaux acteurs éducatifs, aussi bien à l'école qu'à l'extérieur de ce milieu, et ce, afin de favoriser l'adoption d'une conception élargie de l'acte d'enseigner (Gouvernement du Québec, 2020). Cette culture d'entraide et d'ouverture s'appuie sur les influences de divers milieux: culturels, médiatiques, professionnels ou scientifiques (Sanders, 2019). En ce sens, Portelance et ses collaborateurs recommandaient en 2008, et plus récemment en 2019, de mettre en place des actions concertées dès la formation initiale, dans les différents programmes en enseignement afin de soutenir le professionnalisme collaboratif des enseignants.

\section{Des recherches en faveur de la collaboration université-milieu}

Les invitations à intégrer davantage de collaboration universitémilieu pour faire face aux impératifs et défis d'aujourd'hui et de demain se font donc de plus en plus nombreuses provenant à la fois des étudiantes et des étudiants inscrits dans les parcours de formation, des différents intervenants clés qui les accompagnent et des résultats de recherche portant sur le sujet. En effet, l'idée de mettre en place des stratégies collaboratives université-milieu est soutenue par plusieurs recherches en éducation portant précisément sur cet objet (Caron et Portelance, 2017 ; Cody, 2013 ; Correa Molina et Gervais, 2008; Coulombe et al., 2019; Dufour et al., 2018; Leroux et Portelance, 2018; Malo, 2018 ; Morales Perleza, 2016 ; Portelance, Martineau et Presseau, 2008). La plupart de ces travaux soutiennent notamment que le développement des compétences en enseignement serait favorisé par un arrimage soutenu entre les milieux universitaire et scolaire. Cet arrimage passe notamment par des stratégies collaboratives pour accompagner les étudiants en 


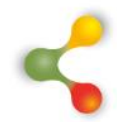

\section{REVUE HYBRIDE DE L'ÉDUCATION}

enseignement (Cody et al., 2019a) et les intervenants en stage ${ }^{1}$ (Cody et al., 2019a ; Gagnon, 2019 ; Portelance et al., 2019 ; Van Nieuwenhoven et Colognesi, 2013).

Dès lors, l'idée de moyens ou de stratégies collaboratives efficientes pour soutenir l'accompagnement des futurs enseignants émerge. Des travaux ont déjà permis d'en identifier certaines pour favoriser l'arrimage des liens théorie-pratique, soutenir les étudiants dans leur formation et les accompagner dans les différentes étapes de l'insertion professionnelle (Cody et al., 2019b; Rivard et al., 2017). Parmi ces stratégies,

- certaines concernent des dispositifs d'accompagnement par mentorat (tutorat par des pairs, des professeurs, des conseillers pédagogiques ou des enseignants);

- d'autres portent sur des possibilités d'échanges d'informations et d'autoformation (artéfacts numériques de contenus de cours, de problèmes ou de solutions expérimentés en stage, d'informations sur les processus d'embauche, sur la réalité du milieu scolaire et sur les exigences liées aux préparatifs d'une rentrée scolaire);

- d'autres renvoient à la réalisation d'activités de coformation (communautés de pratique, communautés d'apprentissage professionnelles en ligne, groupes de codéveloppement professionnel, etc.) ;

- d'autres sur le développement, la création ou l'offre de formations non créditées et créditées.

Ainsi, il s'avère fort pertinent d'élaborer et de mettre en œuvre un dispositif d'accompagnement pour soutenir les futures et nouvelles personnes enseignantes, et ce, peu importe l'ordre d'enseignement ou la discipline. Ce dernier devrait contribuer à soutenir l'émergence et la consolidation des compétences du $21^{\mathrm{e}}$ siècle chez les futurs et nouveaux enseignants pour faciliter leur insertion professionnelle, et ce, en s'appuyant sur la collaboration entre les milieux et les acteurs comme levier pour l'accompagnement.

Concevoir les différentes modalités d'un tel dispositif, dans une perspective de collaboration entre différents milieux et différents intervenants, ne va pas de soi. Cet article se positionne comme une réflexion autour de ces enjeux à travers l'expérience de conception et de mise en œuvre d'une plateforme numérique pour accompagner les futurs enseignants et les nouveaux diplômés dans le développement de leurs compétences, tant en milieu scolaire qu'en milieu universitaire.

\footnotetext{
${ }^{1}$ Ces intervenants réfèrent aux enseignants associés et aux superviseurs universitaires.
} 


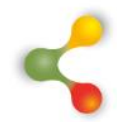

\section{REVUE HYBRIDE DE L'ÉDUCATION}

\section{Cadre théorique}

La notion de collaboration et les défis qui l'accompagnent constituent les fondements de ce récit d'expérience. La présente section propose notre positionnement théorique face à ceux-ci.

La collaboration s'inscrit au cœur de l'élaboration de ce projet. Collaborer suppose «une dynamique interactionnelle plus ou moins intense qui se tisse entre des personnes et qui tient comme idéal une culture collaborative où les personnes travaillent en cohésion, autour d'un but commun, dans des relations d'interdépendance " (Corriveau et Savoie Zajc, 2010, p. 9). Larivée et al. (2017) précise que « la collaboration est de l'ordre de la relation entre des personnes et de la participation à quelque chose au regard d'un objet ou but commun » (p. 13). Cody, Coulombe, Giroux, Gauthier et Gaudreault (2016), à l'instar de Borges (2011), de Dionne et Savoie-Zajc (2011), de Marcel, Dupriez et Bagnoud (2007) et de Portelance, Pharand et Borges (2011), définissent la collaboration comme un lieu pour amorcer un travail conjoint, dans lequel au moins deux individus interagissent et œuvrent collectivement à ce travail. Aussi, selon Cody et al. (2016), la collaboration s'inscrit dans un milieu structuré par une coordination administrative et pédagogique, et constitue un processus complexe renvoyant à un lieu commun (Dionne et Savoie-Zajc, 2011), à un engagement vers un but commun et à une ouverture aux savoirs et aux expériences des autres (Portelance et al., 2011). Autrement posé, la collaboration offre en fait un espace pour travailler ensemble, pour partager, réfléchir et produire autour d'un objectif commun.

Cependant, les écrits nous apprennent également que le travail en collaboration revêt de nombreux défis pour les acteurs. Quatre grandes catégories de défis semblent regrouper un ensemble de manifestations de ces difficultés qui doivent être surmontées pour établir une réelle relation de collaboration (Yuan et Lee, 2015 ; Yuan et Mak, 2016). Le premier défi, nommé «le mur invisible » concerne ce que Beaumont, Lavoie et Couture (2010) considèrent comme les facteurs humains. II réfère notamment à la capacité d'établir une relation de proximité (Gagnon et al., 2015) et un climat favorable aux échanges (Borthwick et al., 2003). L'image du mur illustre la difficulté d'aller vers l'autre, notamment entre les acteurs du milieu de la recherche et ceux du milieu de pratique (Beaumont et al., 2010), dans une relation symétrique plutôt que hiérarchique (Gagnon et al., 2015).

Le deuxième défi réfère au soutien humain et financier du projet collaboratif (Borthwick et al., 2003; Yuan et Lee, 2015). Ce défi se manifeste notamment sous la forme d'un manque de valorisation et de reconnaissance perçu par les acteurs, ainsi que d'un engagement limité de leur part. II s'agit aussi du problème récurrent du financement des activités (Corbin et al., 2017 ; Gagnon et al., 2015). 


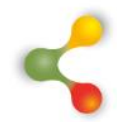

\section{REVUE HYBRIDE DE L'ÉDUCATION}

Le troisième défi relevé dans les écrits concernant la collaboration se rapporte à l'organisation du travail, soit le partage des rôles, des responsabilités et des ressources (Beaumont et al., 2010 ; Gagnon et al., 2015). Les difficultés de communication, induites en partie par le premier défi, freinent l'adhésion des acteurs au projet commun, les plaçant littéralement dans un statut d'itinérant (Beaumont et al., 2010). II en résulte une méconnaissance des objectifs, des attentes, des expertises et des rôles de chacun, menant vers une structure hiérarchique plutôt qu'à la collaboration. La mention fréquente au manque de temps et d'énergie constitue également une manifestation de ce défi (Wang et Zhang, 2013 ; Yuan et Lee, 2015).

L'équilibre entre l'autonomie des parties et leur dépendance s'inscrit dans le quatrième défi qu'est la durabilité et la pérennisation de la collaboration (Yuan et Mak, 2016). Lorsqu'un projet réunit les milieux de la recherche et de la pratique, il est possible d'observer une difficulté pour les acteurs à mobiliser les savoirs issus de l'un ou l'autre des milieux et à les réinvestir de manière autonome. En l'occurrence, il y a dépendance d'un groupe envers l'autre, ce qui limite considérablement la collaboration et, surtout, sa persistance dans le temps (Borthwick et al., 2003). Lorsque la collaboration n'est plus aussi structurée par le projet, notamment en raison du manque de financement, lorsqu'il y a perte d'expertise, les acteurs ont peu de chance de poursuivre la collaboration sur une longue durée (Gagnon et al., 2015).

Ainsi, les prochaines sections décrivent la mise en œuvre d'un dispositif d'accompagnement axé sur la collaboration et destiné aux étudiants en enseignement de partout au Québec, et ce, afin d'incarner les défis propres à la collaboration rencontrés dans le cadre de ce projet. S'ensuivra une discussion autour des stratégies collaboratives mises de l'avant afin de surmonter ses défis.

\section{Méthodologie - Récit d'expérience en théorie}

Dans cet article, le propos se centre non pas sur les résultats d'une étude, mais sur une démarche de mise en œuvre d'un dispositif axé sur la collaboration entreprise par une équipe composée de partenaires interuniversitaires, dont I'UQAC, I'UQAR, I'UQAT, I'UQTR, I'UQO et I'Université de Sherbrooke et de partenaires provenant des régions où sont géographiquement localisées les universités engagées dans ce projet.

Les descriptions présentées dans la section suivante prennent la forme d'un récit d'expérience. Cette méthode, comme le récit de vie ou le récit de pratique (Bertaux, 1980, 1997 ; Desgagné, 2005), s'inscrit dans une perspective autobiographique. Comme le mentionne Balleux (2007), en référence à Pineau et Legrand (2002), c'est la part relative qu'occupe le sujet par rapport aux récits, dans le cas présent un collectif de sujets, qui 


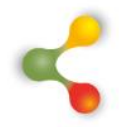

\section{REVUE HYBRIDE DE L'ÉDUCATION}

détermine la perspective. Ainsi, dans le modèle autobiographique, c'est le sujet qui procède à sa propre interprétation, en contraste du modèle biographique où le chercheur mène l'analyse sur le propos rapporté par le sujet et du modèle dialogique dans lequel le travail d'analyse se fait conjointement. Pour reprendre les mots d'Astier, qui réfère à Ricœur (1983), « un tel discours n'est ni celui du souvenir ou du témoignage, ni celui du récit des actions [...] » (2004, p. 39), le récit d'expérience est un genre discursif spécifique empreint de subjectivité, qui repose sur l'affirmation du sujet engagé dans l'action, qui vise à agir sur celui à qui il s'adresse, propose des connaissances transférables tirées de l'action et dont les éléments relatés sont porteurs pour un discours futur.

La production du récit d'expérience qui suit s'est inspirée de la démarche autobiographique proposée par Desmarais et Simon (2007), alliant récit oral et écrit, production individuelle et collectivisation. Préalablement à toute activité restitutrice, il convient de rappeler que l'équipe travaille en étroite collaboration depuis 18 mois, ce qui a permis d'instaurer une dynamique de groupe au sein des membres et un partage des interprétations sur les finalités et les modalités du collectif. En deuxième lieu, il y a eu partage des repères théoriques guidant le projet, notamment le concept de collaboration et les défis qui y sont liés afin d'assurer une compréhension commune de l'objet ${ }^{2}$. Plutôt que de miser sur une présentation orale de récits individuels, comme recommandé par Desmarais et Simon (2007), l'équipe a plutôt opté pour la rédaction de synthèses de rencontres et la tenue d'un tableau planificateur qui ont fait office de journal de bord (Baribeau, 2005 ; Valéau et Gardody, 2016), principalement réalisé par la personne à la coordination du projet, depuis le début de la réflexion entourant son élaboration. À partir de ce matériel de base, le processus dialectique, à l'oral et à l'écrit, entre les récits individuels et collectifs, s'est amorcé. Des portions de récits sont entrées en résonance et d'autres ont plutôt suscité la confrontation des perceptions au sein du groupe. C'est cette activité de collectivisation qui permet d'approfondir, de préciser et de compléter le matériel, lequel est réinterprété à la lumière du cadre théorique et fait l'objet d'une analyse globale par le groupe pour en dégager les principaux éléments porteurs de sens et pertinents à partager. Ce qui a finalement mené à l'activité restitutrice de rédaction du récit d'expérience qui suit.

\footnotetext{
2 En septembre 2018, l'équipe de recherche a tenu une rencontre de validation des interprétations sur les concepts de collaboration et de défis, en plus de déterminer des stratégies collaboratives potentielles pour surmonter ces défis. En mars 2019, un atelier regroupant 40 intervenants du milieu scolaire a été réalisé sur le thème de la collaboration, de ses défis et des stratégies collaboratives à mettre en œuvre.
} 


\section{$\&$}

\section{REVUE HYBRIDE DE L'ÉDUCATION}

\section{Récit de la création d'une plateforme numérique}

Dans la foulée des mandats stratégiques en éducation (concours 2019), une équipe interuniversitaire chapeautée par des chercheuses en éducation de I'UQAC, a mis sur pied un projet de développement d'une plateforme numérique destiné aux futurs et nouveaux diplômés de tous les secteurs d'éducation et d'enseignement (préscolaire, primaire, secondaire, adaptation scolaire, professionnel et adulte) en processus de transition identitaire et professionnelle, nommément TrEnsForma (TRansition pour les ENSeignants et les enseignantes en FORMAtion). Poursuivant, deux objectifs spécifiques: 1) soutenir l'arrimage entre les savoirs théoriques et les savoirs expérientiels et 2) résoudre des situations problématiques vécues en contexte de formation initiale (cours et stages) et en contexte d'insertion professionnelle (0-5 ans), TrEnsForma met à la disposition des futurs et nouveaux enseignants, par le truchement d'une plateforme numérique, diverses modalités d'accompagnement :

- Le mentorat (service de première ligne, mise en relation entre un mentor et un étudiant souhaitant être accompagné) ;

- L'autoformation (bibliothèque virtuelle contenant des ressources pertinentes en lien avec la profession enseignante) ;

- La coformation (mise en place de groupes de codéveloppement, de groupes de discussion à visée pédagogique, de communautés de pratique portant sur différentes thématiques et autres types de dispositifs de même nature) ;

- Les ressources audiovisuelles (conférences, ateliers, colloques, capsules audios et vidéos, etc.) ;

- Les formations créditées (microprogramme de $2^{\mathrm{e}}$ cycle).

Comme mentionné antérieurement, ces modalités d'accompagnement sont structurées de manière à soutenir l'émergence et la consolidation des compétences du $21^{\text {e }}$ siècle chez les futures et nouvelles personnes enseignantes, et ce, en s'appuyant sur la collaboration entre les milieux et les acteurs comme levier pour l'accompagnement.

Afin de saisir les défis posés par l'élaboration de TrEnsForma et les différents enjeux de la collaboration inhérente, nous reprenons ici les grandes étapes et activités qui ont été nécessaires pour en assurer l'édification et, subséquemment, la mise en œuvre. Rétrospectivement, les premières étapes franchies par l'équipe ont été surtout de nature administrative et législative. En fait, il a été a priori nécessaire de se familiariser et de s'intégrer au fonctionnement de l'institution d'attache du projet et de collaborer avec les différentes parties prenantes. Des approches et de nombreuses rencontres ont été organisées en ce sens 


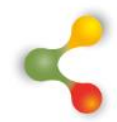

\section{REVUE HYBRIDE DE L'ÉDUCATION}

avec différents services administratifs de l'UQAC ${ }^{3}$, notamment le Service des finances (p. ex. suivi budgétaire) ainsi que celui des ressources humaines (p. ex. embauche de personnel). Également, le projet a impliqué la ratification de documents légaux entre les parties prenantes, notamment entre le ministère de l'Éducation et de l'Enseignement supérieur (MEES), I'UQAC et les universités partenaires ( $p$. ex. convention d'aide financière, ententes de transfert). Ces tâches ont nécessité des itérations nombreuses entre les responsables de TrEnsForma et le secrétariat général de l'UQAC, qui prend habituellement en charge les questions d'ordre juridique. Gérer l'ensemble de ces questions s'est donc avéré une tâche particulièrement chronophage, parfois fastidieuse, qui a occupé les cinq premiers mois du projet (septembre 2019 à janvier 2020) et qui encore aujourd'hui nécessite d'y revenir hebdomadairement. Le caractère « inédit » du projet a été en ce sens un défi supplémentaire pour les responsables qui se sont butées couramment à des demandes semblant n'avoir aucun " précédent » pour les services administratifs, ce qui donnait en quelque sorte l'impression qu'un peu tout le monde apprenait la marche à suivre au même moment. L'enjeu d'avoir accès aux ressources financières et humaines étant néanmoins une condition d'opération sine qua non, il fallut, pour manœuvrer "rapidement », établir une collaboration fonctionnelle avec l'ensemble de ces services.

Une fois plusieurs de ces questions administratives et juridiques répondues, il a été ensuite possible pour l'équipe de s'attaquer à la création de la plateforme ${ }^{4}$, et ce, tant du point de vue de la forme (p. ex. les modalités de fonctionnement des activités d'accompagnement, l'implémentation des activités à l'intérieur de l'infrastructure Web) que du contenu (p. ex. l'élaboration des contenus). Pour ce faire, il a fallu rapidement "activer » les liens entre les partenaires internes à l'UQAC, régionaux et interuniversitaires du projet.

À l'échelle de l'UQAC et régionale, il s'agissait de rencontres et de communications avec les responsables de baccalauréats en enseignement ainsi qu'avec les partenaires des centres de service scolaires ( $p$. ex. conseillers pédagogiques, superviseurs de stage, directions d'établissements scolaires) pour les informer et les impliquer dans la concrétisation du projet et de ses activités. Des rencontres du même ordre ont été organisées avec les partenaires régionaux et internes parmi la plupart des universités associées au projet. Celles-ci avaient pour but de soutenir leur intérêt afin qu'ils demeurent engagés dans la démarche, et ce,

\footnotetext{
${ }^{3}$ Rappelons que l'UQAC est l'université agissant comme « noyau » pour le projet TrEnsForma, c'est-à-dire que l'équipe en place coordonne les activités et assure la reddition de comptes auprès du MEES.

${ }^{4}$ La phase de développement a été la plus touchée par la crise sanitaire. En effet, la gestion de la crise en tant que telle, notamment la migration vers un mode de fonctionnement entièrement numérique, entraina avec elle son lot de défis ( $p$. ex. révision du calendrier de réalisation, démarches auprès du MEES pour obtenir une prolongation, accès difficile aux milieux et intervenants scolaires, etc.) qui pourrait sans doute occuper le propos d'un article à part entière. Nous nous limiterons cependant aux effets les plus directs et immédiats.
} 


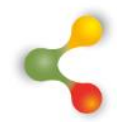

\section{REVUE HYBRIDE DE L'ÉDUCATION}

même dans une partie où leur apport était moins sollicité. II importe toutefois de mentionner que l'accès aux milieux scolaires et aux partenaires a été significativement entravé par la crise sanitaire, nécessitant une migration de l'ensemble des rencontres et, plus largement, des activités vers le numérique. Sans que cela ait été intentionnellement planifié, le passage au numérique s'est révélé un levier à différents égards, comme pour la gestion des communications. Ainsi, l'ensemble de ces échanges avec les partenaires a permis la création d'un réseau, lequel constitue une grande richesse pour alimenter les activités d'accompagnement et rejoindre des utilisateurs potentiels à l'intérieur des milieux scolaires.

Sur le plan interuniversitaire, il s'agissait plus spécifiquement de rencontres avec les autres coresponsables ${ }^{5}$. Ces rencontres ont permis de définir les modalités de participation de chaque institution à l'intérieur du projet, de s'entendre sur une dynamique d'équipe, de définir les besoins financiers et humains de chacun, etc. Un des nombreux défis du projet réside dans l'agencement de la participation des universités, car si tous souhaitaient faire partie du projet, la manière de s'y impliquer est forte différente d'une université à l'autre. Dès lors, afin d'éviter une participation à (trop grande) géométrie variable, il s'est avéré stratégique et nécessaire de déléguer une partie du leadership aux autres co-responsables afin de se pencher sur la définition et le développement des activités d'accompagnement. Des sous-équipes de travail ont ainsi été formées en fonction de l'affinité relative ou de l'intérêt des participants envers le type d'activité pris en charge. Chaque équipe s'est dotée de responsables et d'un échéancier de travail particulier. Pour nourrir la réflexion, deux sondages et un groupe de discussion ont été réalisés auprès des futurs utilisateurs de la plateforme afin de connaître leurs besoins perçus et les problèmes rencontrés, ainsi que les solutions que TrEnsForma pouvait apporter. Les résultats de ces consultations ont eu une grande influence sur le développement des activités et des modalités de mise en œuvre.

Sur le plan technique, il a été nécessaire de se mailler avec un autre partenaire détenant l'expertise pour assurer le développement Web de la plateforme. Une équipe du Centre de soutien à la prestation virtuelle de l'UQAC a été mandatée pour faire la conception de l'interface Web ainsi que des différents éléments graphiques de TrEnsForma. II a donc fallu établir une courroie de transmission efficace entre l'idéation des activités (les sous-groupes) et le développement Web. Évidemment, le développement de la plateforme Web a été un processus de longue haleine qui s'est vu complexifié et retardé par la crise sanitaire, l'équipe de développement étant dans l'urgence affectée à d'autres tâches. Cette phase de développement s'est dès lors étendue sur près d'un an, soit de l'hiver 2020 et à l'hiver $2021^{6}$. À un certain point, il est apparu nécessaire

\footnotetext{
${ }^{5}$ Des responsables ont été désignés pour chaque université. Ces responsables ont pour fonction d'assurer le suivi des informations avec les partenaires locaux et collaborateurs.

${ }^{6}$ La plateforme sera mise à l'essai à partir de mars 2021.
} 


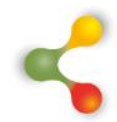

\section{REVUE HYBRIDE DE L'ÉDUCATION}

d'intégrer de nouvelles ressources à l'équipe du projet afin de prendre le relais de l'équipe de développement. Ces ressources prennent désormais en charge le développement graphique ( $p$. ex. montage, facture visuelle des capsules Web) ainsi que l'intégration Web (p. ex. dépôt des capsules Web sur le site, édition de texte des contenus qui sont déposés ou diffusés sur la plateforme).

D'autres démarches ont parallèlement ponctué cette dernière période. En effet, force est de constater que TrEnsForma peut constituer un précieux outil d'accompagnement pour la formation initiale ou l'insertion professionnelle en enseignement, mais revêt aussi un potentiel indéniable pour étudier certaines thématiques touchant la recherche en éducation. Afin de tirer profit de cette richesse, des discussions auprès du comité d'éthique de la recherche de I'UQAC ont été conduites afin de mettre sur pied une banque de données numériques. Évidemment, cela a nécessité de se familiariser avec les procédures et politiques en matière de gestion de données et de produire un cadre de gestion des données pour le projet. Enfin, le pilotage de TrEnsForma a impliqué de nombreuses représentations nationales auprès de différents comités/tables de gestion provinciale (p. ex. Table MELS-Université, Réseau de l'Université du Québec). L'enjeu du maintien des activités et du financement a été soulevé lors de ces rencontres afin de trouver une voie durable à TrEnsForma, lui permettant de survivre à l'épreuve du temps. L'issue de cette démarche n'est pas encore connue.

\section{Discussion}

Les quatre défis liés à la collaboration (le mur invisible, le soutien humain et financier, l'organisation du travail, la durabilité et la pérennisation) ont servi de balises lors de l'élaboration et de la mise en œuvre du dispositif d'accompagnement TrEnsForma. Pour surmonter ces défis et assurer l'instauration et le maintien d'une culture de collaboration visant à favoriser l'engagement de l'équipe et des partenaires ainsi que le professionnalisme collaboratif chez les futurs et nouveaux enseignants, des stratégies collaboratives ont été déployées. À l'instar des défis, nous en retenons quatre types.

\section{Les stratégies collaboratives de communication}

Dans la perspective d'établir une relation symétrique entre les membres de l'équipe issus de différents milieux (Miller et Hafner, 2008), notamment ceux de la recherche et de la pratique, bien que les frontières ne soient jamais très clairement définies, l'équipe a choisi de miser sur une communication transparente et démocratique. À travers cette communication fréquente, il s'est avéré essentiel de laisser un réel pouvoir d'initiative, de négociation et de décision aux personnes qui ont souhaité s'engager (Beaumont et al., 2010 ; Corbin et al., 2017). Plus encore, des rencontres à distance synchrones et en présence ont constitué le point 


\section{6}

\section{REVUE HYBRIDE DE L'ÉDUCATION}

d'ancrage pour la négociation des besoins des différentes constituantes et pour le développement d'un langage commun (vision, buts, objectifs, services, etc.) autour des activités de TrEnsForma (Borthwick, 2003; Gagnon et al., 2015). II apparaît que les stratégies collaboratives de communication constituent le noyau autour duquel se construit la culture de collaboration. Dans ce projet, le pari a été fait qu'en mettant en place une collaboration saine et vivante au sein de l'équipe responsable et de l'équipe de collaborateurs élargie, les personnes accompagnées, de futurs et de nouveaux enseignants de tout ordre d'enseignement, seraient amenées à développer leur propre compétence de collaboration, ce qui, de notre point de vue, fait écho à la proposition des compétences du $21^{\mathrm{e}}$ siècle (Portelance et al., 2008, Portelance et al., 2019).

\section{Les stratégies collaboratives de partage}

Parmi les autres stratégies préconisées, après les stratégies collaboratives de communication qui ont occupé une place cruciale dès que les réflexions autour du projet ont émergé, celles de partage s'avèrent particulièrement importantes. Les stratégies collaboratives de partage réfèrent au défi d'organisation du travail et de partage des rôles, des responsabilités et des ressources (Beaumont et al., 2010 ; Gagnon et al., 2015). Comme mentionné dans notre récit d'expérience, la structuration et l'élaboration des normes administratives, légales et éthiques de même que des procédures diverses ont exigé un travail conséquent. Comme le soutiennent Miller et Hafner (2008), ces stratégies permettent de clarifier les rôles et les limites d'action ainsi que de mutualiser les attentes, sans toutefois viser l'égalité dans la participation, mais en établissant explicitement les rapports entre les individus. Ces éléments ont demandé beaucoup de temps lors du démarrage du projet, mais il convient d'y revenir régulièrement afin de les faire évoluer et de s'assurer de leur applicabilité dans le temps, car c'est sur cette base que s'érigent d'autres stratégies collaboratives de partage comme le travail de coconstruction des ressources mutuelles autour de l'objectif commun (Grudnoff et al., 2017). Ces aspects d'organisation, plus ou moins formels, sont inhérents au monde du travail et en les rendant accessibles, du moins en partie à travers la plateforme numérique de TrEnsForma, l'équipe souhaite sensibiliser les personnes accompagnées à l'importance du partage des ressources, des expertises, des connaissances et des responsabilités dans la profession enseignante.

\section{Les stratégies collaboratives de soutien}

Différentes stratégies de soutien ont également été mises en place par les responsables du projet. Ce soutien a pris la forme d'une personne mandatée à la coordination du projet et dont le rôle consiste à s'assurer de la compréhension des tâches à effectuer, du travail déjà réalisé et des retombées potentielles. En plus du soutien humain, la subvention obtenue a permis de soutenir financièrement les membres collaborateurs des 


\section{$\&$}

\section{REVUE HYBRIDE DE L'ÉDUCATION}

différentes constituantes universitaires en appuyant diverses initiatives inscrites dans les multiples volets de TrEnsForma telles que des journées sur l'insertion professionnelle, un colloque sur les spécialités en enseignement, des groupes de codéveloppement à l'intérieur de cours en formation initiale, etc. Le soutien humain et financier (Gagnon et al., 2015 ; Yuan et Lee, 2015) passe inévitablement par les stratégies collaboratives de communication, ce qui montre l'influence mutuelle des différentes stratégies déployées et rappelle l'importance d'un cadre de référence pour mettre en place des stratégies variées et complémentaires. En soutenant humainement les responsables d'initiatives, notamment en identifiant un intervenant clé pour favoriser les échanges ainsi qu'en régulant une part des activités par la gestion des fonds permettant leur déploiement, nous considérons avoir pu maintenir l'orientation du dispositif autour du développement des compétences de collaboration, autant celles du $21^{\mathrm{e}}$ siècle que celles exposées dans les référentiels des compétences en enseignement.

\section{Les stratégies collaboratives de pérennisation}

Cette dernière catégorie de stratégies touche principalement les prospectives du projet. À ce stade, le renouvellement du soutien financier constitue une préoccupation importante, comme dans la plupart des projets misant sur la collaboration (Borthwick, 2003 ; Corbin et al., 2017 ; Gagnon et al., 2015). Dans cet esprit, les modalités du dispositif sont réfléchies afin d'engager des coûts minimums et des démarches devront être entreprises afin d'obtenir l'engagement de partenaires pour assurer le financement des services offerts. Par ailleurs, des stratégies collaboratives de pérennisation des relations et des partenariats sont déjà établies. En s'inspirant des partenariats en recherche (Yuan et Mak, 2016), l'équipe s'investit stratégiquement et de manière flexible, afin de s'adapter au plus près des besoins des futurs et nouveaux diplômés ainsi que des milieux scolaires (Gagnon et al., 2015). Le contexte de la pandémie de la COVID-19, avec l'implantation massive de l'enseignement en ligne, montre que les besoins évoluent et que le travail enseignant se transforme (Karsenti, 2019 ; Müller et Goldenberg, 2020). Les compétences du $21^{\mathrm{e}}$ siècle s'avèrent impératives pour favoriser la persévérance des nouveaux et des futurs enseignants; de ce fait, l'équipe et les collaborateurs doivent s'ajuster. L'équipe se donne enfin comme priorité de reconnaître et d'encadrer le travail des collaborateurs, chercheurs, praticiens et étudiants afin qu'ils renforcent leurs propres compétences de collaboration, et ce, par des activités intra et interuniversitaires fréquentes.

\section{Conclusion}

Les compétences du $21^{\mathrm{e}}$ siècle constituent des enjeux de formation qui doivent davantage s'inscrire au cœur de la préparation des enseignants d'aujourd'hui et de demain. Parmi ces dernières, la collaboration nous apparaît comme une voie incontournable pour 


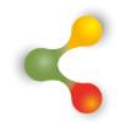

\section{REVUE HYBRIDE DE L'ÉDUCATION}

accompagner les futurs et les nouveaux enseignants dans leur insertion professionnelle. C'est donc sur la collaboration, abordée par les C21 et les référentiels de compétences, que mise justement le projet TrEnsForma. À travers un récit d'expérience, cet article a mis en lumière les (nombreux) défis qu'ont posés l'élaboration et dans une moindre mesure, l'opérationnalisation d'une plateforme numérique, des défis souvent inhérents à toute initiative s'appuyant sur la collaboration entre les acteurs et les milieux éducatifs. En outre, la description de ces défis a aussi permis de dégager diverses stratégies collaboratives qui se sont avérées des leviers à la concrétisation du projet. En effet, à la lumière des actions posées jusqu'à présent et celles à venir, il apparaît que les principaux défis de la collaboration peuvent être relevés en misant, en premier lieu, sur la communication, une stratégie collaborative essentielle à l'établissement du réseau de partenaires qui constitue également le socle sur lequel s'appuient les autres stratégies déployées. En effet, la communication demeure un élément clé du partage des rôles, des responsables et des ressources, qu'elles soient humaines, financières, matérielles ou autres, au sein du projet. La communication est également à la base du soutien, principalement le soutien humain, apporté aux membres du projet, en plus de permettre les négociations en ce qui concerne les contributions au fonctionnement du projet et les utilisations du financement.

Par ailleurs, à l'aube d'une phase plus intensive de déploiement de la plateforme, nos réflexions se tournent désormais vers les étapes et les défis qui jalonneront l'opérationnalisation de l'accompagnement auprès des utilisateurs-étudiants. Forte des expériences acquises lors de la première phase, l'équipe souhaite évidemment compter sur la collaboration pour, entre autres, alimenter la plateforme par la création en continu de ressources éducatives (artéfacts) ainsi que pour maintenir les activités d'accompagnement (mentorat, communautés de pratique, groupes de codéveloppement, etc.). Entre autres, l'activité mentorale permettra de saisir les besoins des futurs et nouveaux enseignants, orientant la recension et la production de ressources ainsi que le développement de l'expertise nécessaire à l'accompagnement des personnes en insertion professionnelle, autant pendant leur formation qu'à leur entrée dans la carrière.

Enfin, à l'approche de l'échéance du financement actuel, force est de constater que les stratégies collaboratives de pérennisation occuperont une place de plus en plus importante au sein des travaux de l'équipe. En effet, plusieurs ressources sont présentement mises à contribution et de grands efforts sont investis pour l'élaboration de la plateforme, mais il importe de ne pas perdre de vue que ceux-ci pourraient s'avérer vains advenant l'impossibilité de lui assurer un avenir pérenne. À la différence des stratégies collaboratives de communication, de partage et de soutien, celles de pérennisation échappent d'une certaine manière au contrôle des équipes derrière les initiatives et les projets. Alors qu'elles sont en mesure d'ajuster leurs stratégies de communication, qu'elles peuvent renégocier le 


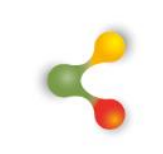

\section{REVUE HYBRIDE DE L'ÉDUCATION}

partage des ressources, des rôles et des responsabilités et qu'elles ont le pouvoir d'ajouter des moyens pour soutenir les collaborateurs pendant les phases d'élaboration et de mise en œuvre des projets, le maintien du financement permettant d'assurer sa durabilité dans le temps ne leur appartient pas. Certes, l'équipe de TrEnsforma multipliera les démarches auprès des instances concernées, notamment les universités partenaires et le MEES, mais cela demeure, comme pour toutes initiatives financées par un budget ponctuel, un enjeu majeur. 


\section{REVUE HYBRIDE DE L'ÉDUCATION}

\section{Références}

Anderson, J. (2010). ITC transforming education: a regional guide. UNESCO Bangkok.

Astier, P. (2004). Parler d'expérience. Formation emploi, 88(1), 33-42.

Balleux, A. (2007). Le récit phénoménologique : étape marquante dans l'analyse des données. Recherches qualitatives, 3, 396-423.

Baribeau, C. (2005). Le journal de bord du chercheur. L'instrumentation dans la collecte des données, Hors Série(1), 98-114.

Beaumont, C., Lavoie, J. et Couture, C. (2010). Les pratiques collaboratives en milieu scolaire : cadre de référence pour soutenir la formation. Université Laval (CRIRES).

Bertaux, D. (1980). L'approche biographique, sa validité méthodologique, ses potentialités. Cahiers internationaux de sociologie, LXIX(2), 198225.

Bertaux, D. (1997). Les récits de vie. Nathan.

Borges, C. (2011). La collaboration enseignante en éducation physique et à la santé. Dans L. Portelance, C. Borges et J. Pharand (dir.), La collaboration dans le milieu de l'éducation (p. 83-102). Presses de l'Université du Québec.

Borthwick, A. C., Stirling, T., Nauman, A. D. et Cook, D. L. (2003). Achieving successful school-university collaboration. Urban Education, 38(3), 330-371.

Bryan, L. A., Moore, T. J., Johnson, C. C. et Roehrig, G. H. (2015). Integrated STEM Education. Dans C. C. Johnson, E. E. PetersBurton et T. J. Moore (dir.), STEM Road Map : A Framework for Integrated STEM Education (p. 23-37). Routledge.

Caron, J. et L. Portelance (2017). La collaboration entre chercheure et praticiens dans un groupe de codéveloppement professionnel. Éducation et Socialisation, https://doaj.org/article/fd76ed4e4e9a42ecabd99cb901e3ce08?frbrV ersion $=2$

Cody, N., Coulombe, S., Doucet, M., Gagné, A. et Labrecque, R. (2019, avril). Des stratégies collaboratives université-milieu scolaire pour soutenir l'alternance en formation initiale à l'enseignement [présentation orale]. Enjeux actuels et futurs de la formation et de la profession enseignante, Montréal, QC, Canada. 


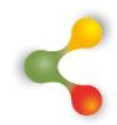

\section{REVUE HYBRIDE DE L'ÉDUCATION}

Cody, N., Coulombe, S., Giroux, P., Gauthier, D. et Gaudreault, S. (2016). Pratiques, objets et finalités de collaboration en lien avec l'intégration des tablettes numériques dans une école secondaire. Canadian Journal of Learning and Technologies, 42(3). http://www.cj|t.ca/index.php/cj|t/article/view/27473

Cody, N., Nadeau-Tremblay, S., Boudreault, S., Coulombe, S., Doucet, M, De Champlain, A., Gagné, A., Labrecque, R., Lavoie, A., Leroux, C., Rose, N. St-Gelais, S. et Tremblay, I. (2019, mars). Atelier "Des milieux partenaires pour la formation de la relève en enseignement » [document inédit]. Université du Québec à Chicoutimi.

Corbin, J. H., Chu, M., Carney, J., Donnelly, S. et Andrea, C. (2017). Understanding Collaboration: A Formative Process Evaluation of a State-Funded School-University Partnership. The Journal of the National Association for Professional Development Schools, 10(1), 35-45.

Correa Molina, E. et Gervais, C. (2008). Les stages en formation à l'enseignement. Pratiques et perspectives théoriques. Presses de l'Université du Québec

Coulombe, S., Doucet, M. et Zourhlal, A. (2019). Le codéveloppement professionnel, un dispositif favorisant l'insertion professionnelle des enseignants en formation professionnelle. Dans M. Doucet et M. Thériault (dir.), L'adulte en formation... pour devenir soi. Espaces, passages, débats et défis. Presses de l'Université du Québec.

Desgagné, S. (2005). Récits exemplaires de pratique enseignante: analyse typologique. Presses de l'Université du Québec.

Desmarais, D. et Simon, L. (2007). La démarche autobiographique et son objet: enjeux de production de connaissance et de formation. Recherches qualitatives, 3, 350-370.

Dionne, L. et Savoie Zajc, L. (2011). Sens, caractéristiques et retombées de la collaboration entre enseignants et contribution au développement professionnel. Dans L. Portelance, C. Borges et J. Pharand (dir.), La collaboration dans le milieu de l'éducation: dimensions pratiques et perspectives théoriques (p. 45-60). Presses de l'Université du Québec.

Dufour, F. Portelance, L., Van Nieuwenhoven, C. et Vivegnis, I. (2018). Préparer à l'insertion professionnelle pendant la formation initiale en enseignement. Presses de l'Université du Québec.

Gagnon, C. (2019). L'apport individuel et collectif AU et DU groupe de codéveloppement professionnel : une analyse à travers les différents chapeaux adoptés par des enseignants associés de la formation professionnelle. Dans F. Vandercleyen, M.-J. Dumoulin et M. L'Hostie (dir.), Le groupe de codéveloppement professionnel pour 


\section{$\&$}

\section{REVUE HYBRIDE DE L'ÉDUCATION}

former à l'accompagnement de stagiaires : conditions, enjeux et perspectives (p. 125-153). PUQ.

Gagnon, B., Charron, A. et Raby, C. (2015, Juin). Collaborer et coopérer pour la mise en œuvre de projets de recherche-action: Un partenariat gagnant pour le développement professionnel des enseignants [communication orale]. Biennale internationale de l'Éducation, de la Formation et des Pratiques professionnelles, Paris, France.

Gouvernement de l'Ontario. (2016). Phase 1-Définir les compétences du 21e siècle pour l'Ontario: document de réflexion. Ministère de l'Éducation de l'Ontario. https://edusourceontario.com/content.aspx?name=pedagogie a I ere numerique\&submenu=ressources et references\&id=41\&id su $\underline{\text { bmenu }=200}$

Gouvernement du Québec. (2020). Référentiel de compétences professionnelles : Profession enseignante.

Grudnoff, L., Haigh, M. et Mackisack, V. (2017). Re-envisaging and reinvigorating school-university practicum partnerships, Asia-Pacific Journal of Teacher Education, 45(2), 180-193, DOI: 10.1080/1359866X.2016.1201043

Hart, S. A. et Ouellet, D. (2013). Les compétences du 21e siècle. Bulletin de l'Observatoire des compétences-emplois, 4(4). https://oce.uqam.ca/les-competences-qui-font-consensus/

Karsenti, T. (2019). Le numérique en éducation: Pour développer des compétences. Presses de l'Université du Québec.

Larivée, S. J., Kalubi, J.-C., Larose, F., Couturier, Y., Bédard, J., Lude, P. (2017). Les pratiques de collaboration école-famille-communauté efficaces ou prometteuses : synthèse des connaissances et pistes d'intervention (Rapport de la recherche FRQSC 2015-AP-187774). Université de Montréal. https://frq.gouv.qc.ca/app/uploads/2021/06/ap 20142015 larivees rapport ecole-famille-communaute.pdf.pdf

Leroux, M. et Portelance, L. (2018). Les initiatives du milieu universitaire et du milieu scolaire pour favoriser la préparation à la transition vers l'insertion dans la profession enseignante. Vers une alternance intégrative ? Dans P. Chaubet, M. Leroux, C. Masson, C. Gervais et A. Malo (dir.) Apprendre et enseigner en contexte d'alternance : vers la définition d'un noyau conceptuel (p. 247-271). PUQ.

Malo, A. (2018). Apprendre en stage selon différentes configurations de la situation de travail et de formation. Une étude de cas. Dans $P$. Chaubet, M. Leroux, C. Masson, C. Gervais et A. Malo (dir.) Apprendre et enseigner en contexte d'alternance : vers la définition d'un noyau conceptuel (p. 45-66). PUQ. 


\section{8}

\section{REVUE HYBRIDE DE L'ÉDUCATION}

Marcel, J.- F., Dupriez, V. et Bagnoud, D. P. (2007). Le métier d'enseignant : nouvelles pratiques, nouvelles recherches. Dans J.F. Marcel, V. Dupriez, D. P. Bagnoud et M. Tardif (dir.), Coordonner, collaborer, coopérer: de nouvelles pratiques enseignantes ( $p .7-17)$. De Boeck Université.

Miller, P. et Hafner, M. (2008). Moving Toward Dialogical Collaboration: A Critical Examination of a University-School-Community Partnership. Educational Administration Quarterly, 44(1), 66-110.

Morales Perleza, A. (2016). Les savoirs professionnels à la base de la formation des enseignants au Québec et en Ontario : une étude comparative des modèles universitaires de professionnalisation et de leurs enjeux [thèse de doctorat, Université de Montréal], Papyrus.

Müller, L.-M. et Goldenberg, G. (2020, 3 février). Education in times of crisis: Teachers' views on distance learning and school reopening plans during COVID-19: Analysis of responses from an online survey and focus groups. Chartered College of Teaching. https://my.chartered.college/wpcontent/uploads/2020/07/EducationInTimesOfCrisisll 20200708 fin al.pdf

Portelance, L., Caron, J., et Murray-Dugré, L. (2019). Collaboration à l'organisation des stages La collaboration inhérente à l'organisation des stages en enseignement: points de vue de responsables universitaires. Éducation \& Formation, e-314, 86-96.

Portelance, L., Gervais, C., Lessard, M. et Beaulieu, P. (2008). La formation des enseignants associés et des superviseurs universitaires (Rapport de recherche). Table ministère de l'Éducation, du Loisir et du Sport - Universités (MELS-Universités).

Portelance, L., Martineau, S. et Presseau, A. (2008). Les représentations des finissants en formation à l'enseignement quant à la collaboration avec les acteurs du milieu scolaire au moment de l'entrée dans la profession. Dans L. Portelance, J. Mukamurera, S. Martineau et C. Gervais. L'insertion dans le milieu scolaire. Une phase cruciale du développement professionnel de l'enseignant (p. 125-144). Presses de l'Université Laval.

Portelance, L., Pharand, J. et Borges, C. (2011) Mieux comprendre la collaboration pour mieux collaborer. Dans L. Portelance, C. Borges, et J. Pharand. La collaboration dans le milieu de l'éducation. Dimensions pratiques et perspectives théoriques (p.211-224). Presses de l'Université du Québec.

Pruneau, D., Kerry, J., Langis, J. et Léger, M. (2015). Améliorer les programmes canadiens de sciences et technologies au primaire par l'ajout de compétences du $21^{\mathrm{e}}$ siècle. Canadian Journal of Education, 38(3), $1-23$. http://www.jstor.org/stable/canajeducrevucan.38.3.07 


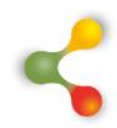

\section{REVUE HYBRIDE DE L'ÉDUCATION}

Ricœur, P. (1983). Temps et récit I. Gallimard.

Rivard, M.- C., Grenier, J., Turcotte, S., Morency, L., Bordeleau, C., Leroux, M. et Marzouk, A. (2017, 18-19 mai). Le parcours d'insertion professionnelle de deux enseignants en ÉPS : facteurs associés à la motivation et à la satisfaction au travail [communication orale]. Colloque CRIFPE, Les défis actuels et futurs de la recherche en enseignement de l'éducation physique et à la santé, Montréal, Qc, Canada.

Sanders, M. G. (2019). The Role of Community in Comprehensive School, Family and Community Programs. Dans R. Deslandes, (dir.), Collaborations école-famille-communauté : recension des écrits (tome 2 : Relations école-communauté), Périscope.

Valéau, P. et Gardody, J. (2016). La communication du journal de bord : un complément d'information pour prouver la vraisemblance et la fiabilité des recherches qualitatives. Recherches qualitatives, 35(1), 76-100.

Van Nieuwenhoven, C. et Colognesi, S. (2013). Une recherche collaborative autour des difficultés des maîtres de stage à accompagner leur stagiaire. Interacçöes, 27, 118-138.

Voogt, J. et Pareja Roblin. N. (2012). A comparative analysis of international frameworks for 21 st Century competences: Implications for national curriculum policies. Journal of Curriculum Studies, 44, 299-321.

Wang, Q. et Zhang, H. (2014). Promoting teacher autonomy through university-school collaborative action research. Language Teaching Research, 18(2), 222-241.

Westera, W. (2001). Competences in education. A confusion of tongues. Journal of Curriculum Studies, 33, 75-88.

Yuan, R. et Lee, I. (2015). Action research facilitated by university-school collaboration. Elt Journal, 69(1), 1-10.

Yuan, R. et Mak, M. (2016). We teach, we record, we edit, and we reflect: Engaging pre-service language teachers in video-based reflective practice. Language Teaching Research, 70(4), 382-391. 